\title{
Preface: Recent advances in Crustacean Genomics
}

\author{
Guiomar Rotllant • Ferran Palero • Peter B. Mather • Heather D. Bracken-Grissom • \\ M. Begoña Santos
}

Received: 4 July 2018/Revised: 14 September 2018/Accepted: 15 September 2018/Published online: 8 November 2018

(C) Springer Nature Switzerland AG 2018

\begin{abstract}
This Special Issue on Crustacean Genomics arises from the TCS 2017 conference held in Barcelona, Spain, between 19 and 22 June, 2017. Applications of next generation sequencing have expanded rapidly over recent years, unveiling many new and exciting areas of genomic research. This includes novel insights into physiology, reproductive biology, response to environmental challenges, and their evolutionary history. Despite their importance, crustaceans still lack genomic resources compared
\end{abstract}

Guiomar Rotllant and Ferran Palero contributed equally to this work.

Guest editors: Guiomar Rotllant, Ferran Palero, Peter Mather, Heather Bracken-Grissom \& Begoña Santos / Crustacean

Genomics

\section{G. Rotllant $(\bowtie)$}

Institut de Ciències del Mar (ICM-CSIC), PasseigMarítim de la Barceloneta 37-49, 08003 Barcelona, Spain

e-mail: guio@icm.csic.es

F. Palero $(\square)$

Centre d'Estudis Avançats de Blanes (CEAB-CSIC), Carrer d'Accés a la Cala Sant Francesc, 14, 17300 Blanes, Spain

e-mail: fpalero@ceab.csic.es

P. B. Mather

Australian Rivers Institute, Griffith University, Nathan,

QLD 4111, Australia with other widely-studied groups such as insects and vertebrates. The present volume integrates a collection of contributions from the speakers, and compiles the main results presented during the symposium. Following the structure of the Crustacean Genomics workshop, contributed papers have been arranged in two main blocks, a first set of studies focused on the use of transcriptomics to investigate crustacean physiology and reproduction while the second set focused on molecular systematics and evolutionary studies.

Keywords Next generation sequencing - Molecular systematics · Growth · Reproduction · Crustacea · Genomics

H. D. Bracken-Grissom

Florida International University, Biscayne Bay Campus, 3000 NE 151st St., MSB 350, North Miami, FL 33181, USA

M. B. Santos

Instituto Español de Oceanografía (IEO), Centro Oceanográfico de Vigo, Sunida a Radio Faro. 50, 36390 Vigo, Spain 


\section{Introduction}

The use of next generation sequencing (NGS) has expanded rapidly over recent years, unveiling in many cases how animal genomes respond to environmental challenges and how they have evolved. Despite the relative importance of crustaceans, notably shrimps, lobsters, crabs, krill, copepods, and barnacles that are familiar to the average person, the first fully assembled and annotated genome developed was that of the water flea, Daphnia pulex. In addition to this valuable resource, there are incomplete genome assemblies from a few species, including two economically important decapod species (Chinese mitten crab, Eriocheir sinensis, and Pacific White Shrimp, Litopenaeus vannamei) (Colbourne et al., 2011; Yu et al., 2015; Song et al., 2016). To address this apparent lack of genomic resources of crustaceans, a special symposium on Crustacean Genomics was organized during the last "The Crustacean Society Mid-Year Meeting 2017", coordinated by scientists from the Spanish Higher Council for Scientific Research (CSIC) and the University of Barcelona (UB). The meeting was held at the Cosmocaixa in Barcelona on 19-22 June. The symposium was arranged in two main blocks, a first set of seminars focused on new developments using transcriptomics to study crustacean physiology and reproduction while a second set focused on systematics and evolutionary studies.

\section{Transcriptomic insights into crustacean physiology and reproduction}

This special issue includes reviews and research papers that highlight the use of NGS approaches to address key questions in reproductive biology and physiology, from discovery of genes responsible for the molting process to analyses of natural selection and adaptation to different salinities, particularly focusing on estuarine and freshwater shrimps. Nguyen et al. (2018, this volume) stress the importance of following specific experimental design guidelines for an adequate gene validation while undertaking transcriptomic studies. For example, it should be taken into account that growth in crustaceans is a discontinuous processes and that repeated shedding of the integument requires formation of a new cuticle and periodic synthesis of structural proteins. Abehsera et al. (2018, this volume) show how changes in morphology are tightly linked to expression patterns of structural proteins. The authors present the moltrelated expression pattern of the gene that encodes for the gastrolith protein (GAP65), a core structural protein involved in cuticle formation. Accurately assigning gene functions is particularly challenging for complex processes like metamorphosis, as it requires a considerable amount of changes to expression patterns in several genes. Ventura et al. (2017, this volume) review current information and provide several examples of how an integrated genomics approach can help to identify the key molecular factors involved in crustacean metamorphosis. A summary of current information on sex determination in decapods is presented by Chandler et al. (2017, this volume) and includes chromosomal mechanisms and "sex-regulator" homologues characterized in model species. Powell et al. (2017, this volume) provide new insights into the molecular mechanisms that explain why, in some crustacean taxa, growth rates in females are faster than in males. Specifically, these authors confirm the increased expression of transcripts with catabolic functions and binding of chitin in females of the banana shrimp (Fenneropenaeus merguiensis).

Rotllant et al. (2017, this volume) identify new candidate genes involved in ovarian development using NGS technologies and highlight how their identification can assist in a better understanding of this process. The main neuroendocrine factors identified belong to the $\mathrm{CHH}$ family of neuropeptides that are produced in the eyestalk. A research paper by Aziz et al. (2018, this volume) investigates another novel aspect of reproduction in certain crustaceans such as the social dominance hierarchy in male giant freshwater prawn (Macrobrachium rosenbergii) that leads to distinct adult male morphotypes varying in size and average weight and that has an impact over the total biomass. The authors suggest a role for opsin (photoreceptive pigment proteins involved in color vision) gene expression in the eyestalk to apparently suppress adult male morph metamorphosis in subordinate males. A comprehensive analysis of differentiallyexpressed genes during maturation of the Norway lobster (Nephrops norvegicus) is also presented by Rotllant et al. (2018, this volume). The authors highlight the need for increasing knowledge on reproduction, specifically at the molecular level, to 
aid the management of a commercially overexploited species.

As illustrated by the studies reported above, assigning gene functions in crustaceans is a difficult task since many genes found are novel, without a direct match in other arthropods. Therefore, additional molecular studies and laboratory experiments will be required to unravel their functions in detail.

\section{Evolutionary biology and systematics}

A major goal in evolutionary biology is to understand the role of adaptive processes on sensory systems. Vision can be crucial for an individual's survival and is often strongly influenced by environmental and ecological factors. Crustaceans utilize a vast array of habitats and ecological niches, and thus provide ideal taxa for investigating the evolution of visual systems. Perez-Moreno et al. (2018, this volume) used a comparative approach to identify and classify opsin genes in two crustaceans (Hyalella azteca and D. pulex). They demonstrate the power and promise of using phylogenetic annotation and a genomic approach for studying sensory systems in both model and non-model organisms. Rahi et al. (2018, this volume) reviewed candidate genes associated with the maintenance of ionic balance in decapods and provide examples of underlying genetic mechanisms involved in adaptation to different environmental salinity gradients. Differentially-expressed genes involved in osmoregulation, temperature tolerance, and reproduction are also identified in two highly divergent lineages of an Australian freshwater shrimp (Rolg et al., 2018, this volume).

Sequencing of the complete mitochondrial genome of Gammarus roeseli, an amphipod species widespread in European continental freshwaters, allowed Cormier et al. (2018, this volume) to infer the evolutionary dynamics of the mitochondrial control region in Amphipoda. These authors identified an unusual case of structural variation in mitochondrial genomes caused by gene duplication. The importance of NGS in population genetics and conservation studies was also highlighted during the symposium. Timm et al. (2018, this volume) used a pilot ddRADseq dataset together with three mitochondrial loci $(12 \mathrm{~S}, 16 \mathrm{~S}$, and $\mathrm{COI})$ to examine population dynamics of the giant isopod (Bathynomus giganteus).
The results obtained suggest that habitat heterogeneity has been the main factor influencing population dynamics in this deep-sea species.

\section{Next steps for Crustacean Genomics}

The nearly 70,000 described species of Crustacea represent one of the largest extant groups in the animal kingdom, and hence contribute a major component to the Earth's biodiversity (Ahyong et al., 2011; Martin et al., 2014). NGS and Third Generation Sequencing provide rapid, cost-efficient technologies for the discovery of genetic markers and new genomic resources, but only recently it has been applied in crustacean biology studies. The wide range of topics covered in this special issue that span from crustacean physiology and reproduction to molecular systematics and evolutionary studies shows the broad spectrum of questions that can be addressed by applying these new technologies. The ongoing decline in experimental costs and the increase in sampling effort of rare taxa will most likely allow scientists to resolve many of the controversies about crustacean phylogenetic relationships. The joint analyses of genomic and transcriptomic data, as shown in several research papers within this special issue, will most surely bring unexpected insights to metabolic and gene expression networks for this fascinating group of animals. These new techniques will also open the possibility for understanding the huge diversity of forms and adaptations observed among crustaceans.

\section{References}

Abehsera, S., S. Weil, R. Manor \& A. Sagi, 2018. The search for proteins involved in the formation of crustacean cuticular structures. Hydrobiologia. https://doi.org/10.1007/s10750018-3684-y.

Ahyong, S. T., J. K. Lowry, M. Alonso, R. N. Bamber, G. A. Boxshall, P. Castro, S. Gerken, G. S. Karaman, J. W. Goy \& D. S. Jones, 2011. Subphylum Crustacea Brünnich, 1772. Zootaxa 3148: 165-191.

Aziz, D., L. Rahi, D. A. Hurwood \& P. B. Mather, 2018. Analysis of candidate gene expression patterns of adult male Macrobrachium rosenbergii morphotypes in response to a social dominance hierarchy. Hydrobiologia. https://doi.org/10.1007/s10750-018-3721-x.

Chandler, J., A. Elizur \& T. Ventura, 2017. The decapod researcher's guide to the galaxy of sex determination. 
Hydrobiologia. https://doi.org/10.1007/s10750-017-34524.

Colbourne, J., M. Pfrender, D. Gilbert, W. Thomas, A. Tucker, T. Oakley, S. Tokishita, A. Aerts, G. Arnold \& M. Basu, 2011. The ecoresponsive genome of Daphnia pulex. Science 331: 555-561.

Cormier, A., R. Wattier, M. Teixeira, T. Rigaud \& R. Cordaux, 2018. The complete mitochondrial genome of Gammarus roeselii (Crustacea, Amphipoda): insights into mitogenome plasticity and evolution. Hydrobiologia. https://doi. org/10.1007/s10750-018-3578-z.

Martin, J. W., J. Olesen, J. T. Høeg \& J. Høeg, 2014. Atlas of Crustacean Larvae. JHU Press, Baltimore.

Nguyen, T. V., H. Jung, G. Rotllant, D. Hurwood, P. Mather \& T. Ventura, 2018. Guidelines for RNA-seq projects: applications and opportunities in non-model decapod crustacean species. Hydrobiologia. https://doi.org/10. 1007/s10750-018-3682-0.

Perez-Moreno, J. L., D. De Leo, F. Palero \& H. D. BrackenGrissom, 2018. Phylogenetic annotation and genomic architecture of opsin genes in Crustacea. Hydrobiologia. https://doi.org/10.1007/s10750-018-3678-9.

Powell, D., A. Elizur \& W. Knibb, 2017. Sex-specific transcript expression in the hepatopancreas of the banana shrimp (Fenneropenaeus merguiensis). Hydrobiologia. https://doi. org/10.1007/s10750-017-3438-2.

Rahi, L., A. Moshtaghi, P. B. Mather \& D. A. Hurwood, 2018. Osmoregulation in decapod crustaceans: physiological 3 and genomic perspectives. Hydrobiologia. https://doi.org/ 10.1007/s10750-018-3690-0.

Rolg, K. A., L. Rahi, J. W. L. Royle, P. J. Prentis \& D. A. Hurwood, 2018. A transcriptome-wide assessment of differentially expressed genes among two highly divergent, yet sympatric, lineages of the freshwater Atyid shrimp,
Paratya australiensis. Hydrobiologia. https://doi.org/10. 1007/s10750-018-3716-7.

Rotllant, G., T. V. Nguyen, J. Aizen, S. Suwansa-ard \& T. Ventura, 2017. Toward the identification of female gonadstimulating factors in crustaceans. Hydrobiologia. https:// doi.org/10.1007/s10750-017-3497-4.

Rotllant, G., T. V. Nguyen, D. Hurwood, V. Sbragaglia, T. Ventura, J. B. Company, S. Joly, A. Elizur \& P. B. Mather, 2018. Evaluation of genes involved in Norway lobster (Nephrops norvegicus) female sexual maturation using transcriptomic analysis. Hydrobiologia. https://doi.org/10. 1007/s10750-018-3521-3.

Song, L., C. Bian, Y. Luo, L. Wang, X. You, J. Li, Y. Qiu, X. Ma, Z. Zhu, L. Ma, Z. Wang, Y. Lei, J. Qiang, H. Li, J. Yu, A. Wong, J. Xu, Q. Shi \& P. Xu, 2016. Draft genome of the Chinese mitten crab, Eriocheir sinensis. GigaScience 5(1): 1-3. https://doi.org/10.1186/s13742-016-0112-y.

Timm, L. E., B. Moahamed, D. Churchill \& H. D. BrackenGrissom, 2018. Bathynomus giganteus (Isopoda: Cirolanidae) and the canyon: a population genetics assessment of De Soto Canyon as a glacial refugium for the giant deepsea isopod. Hydrobiologia. https://doi.org/10.1007/ s10750-018-3563-6.

Ventura, T., F. Palero, G. Rotllant \& Q. P. Fitzgibbon, 2017. Crustacean metamorphosis: an omics perspective. Hydrobiologia. https://doi.org/10.1007/s10750-017-3445-3.

Yu, Y., X. Zhang, J. Yuan, F. Li, X. Chen, Y. Zhao, L. Huang, H. Zheng \& J. Xiang, 2015. Genome survey and high-density genetic map construction provide genomic and genetic resources for the Pacific White Shrimp Litopenaeus vannamei. Scientific Reports 5: 15612. https://doi.org/10. 1038/srep15612.

http://www.nature.com/articles/srep15612\#supplementaryinformation. 